\title{
Elucidating the Site of Protein-ATP Binding by Top-Down Mass Spectrometry
}

\author{
Sheng Yin and Joseph A. Loo ${ }^{\mathrm{a}, \mathrm{b}}$ \\ a Department of Chemistry and Biochemistry, University of California-Los Angeles, Los Angeles, California, USA \\ ${ }^{\mathrm{b}}$ Department of Biological Chemistry, David Geffen School of Medicine, University of California-Los \\ Angeles, Los Angeles, California, USA
}

\begin{abstract}
A Fourier-transform ion cyclotron resonance (FT-ICR) top-down mass spectrometry strategy for determining the adenosine triphosphate (ATP)-binding site on chicken adenylate kinase is described. Noncovalent protein-ligand complexes are readily detected by electrospray ionization mass spectrometry (ESI-MS), but the ability to detect protein-ligand complexes depends on their stability in the gas phase. Previously, we showed that collisionally activated dissociation (CAD) of protein-nucleotide triphosphate complexes yield products from the dissociation of a covalent phosphate bond of the nucleotide with subsequent release of the nucleotide monophosphate (Yin, S. et al., J. Am. Soc. Mass Spectrom. 2008, 19, 1199-1208). The intrinsic stability of electrostatic interactions in the gas phase allows the diphosphate group to remain noncovalently bound to the protein. This feature is exploited to yield positional information on the site of ATP-binding on adenylate kinase. CAD and electron capture dissociation (ECD) of the adenylate kinase-ATP complex generate product ions bearing monoand diphosphate groups from regions previously suggested as the ATP-binding pocket by NMR and crystallographic techniques. Top-down MS may be a viable tool to determine the ATP-binding sites on protein kinases and identify previously unknown protein kinases in a functional proteomics study. (J Am Soc Mass Spectrom 2010, 21, 899-907) (C) 2010 American Society for Mass Spectrometry
\end{abstract}

$\mathrm{P}$ roteins function in biology through direct interaction with other molecules. A ligand can be a molecule, an atom, or an ion that binds to a specific site on the protein by a variety of means, including hydrogen bonding, Van der Waals interactions, and ionic forces. Determining the presence of protein-ligand interactions and the structures of proteinligand complexes are of critical importance in biology, and this knowledge is often essential for efforts to establish new molecular drug targets and to develop more potent medicines.

A number of biophysical tools are available to characterize the interaction between a protein and its ligand(s). However, mass spectrometry (MS) offers potential advantages in sensitivity, specificity, and speed, especially compared with high-resolution nuclear magnetic resonance (NMR) spectroscopy and X-ray crystallography. With electrospray ionization (ESI) to ionize macromolecules without disrupting covalent bonds while maintaining weak noncovalent interactions, the ESI-MS molecular mass measurement provides direct evidence for protein-ligand associations. The proteinligand interactions are often sufficiently retained upon

Address reprint requests to Dr. J. A. Loo, University of California-Los Angeles, Molecular Biology Institute, 402 Paul D. Boyer Hall, 405 Hilgard Avenue, Los Angeles, CA 90095, USA. E-mail: JLoo@chem.ucla.edu the transition from solution to the gas phase that the complex size and binding stoichiometry can be measured [1-3].

Although binding stoichiometry and, in many examples, the relative and absolute solution binding affinities can be measured by ESI-MS methodologies, determining the precise ligand binding site on a target protein is not easily tractable using MS directly. Tandem mass spectrometry (MS/MS) is widely applied to derive the amino acid sequence of small polypeptides (for example, in the so-called bottom-up proteomics approach), and MS/MS of intact proteins (i.e., top-down MS [4]) show potential to be a tool for protein identification and elucidation of post-translational modifications [5]. However, the application of top-down MS to access a protein's primary structure while preserving noncovalent ligand binding would seem to be an insurmountable task because of the lability of the protein-ligand interaction, both in solution and in the gas phase.

However, recent reports suggest that an MS approach may be useful for pinpointing protein-ligand binding. Hydrogen-deuterium exchange (HDX) experiments can yield structural information on the regions of a protein involved in protein-protein and proteinligand contacts [6]. Limited proteolysis of protein complexes can sometimes deliver clues on the sites of ligand binding. For example, we used limited trypsinolysis 
with ESI-MS to determine the sites of zinc- and oligonucleotide-binding to a nucleocapsid zinc finger protein [7]. Our laboratory first demonstrated the application of top-down mass spectrometry, coupled with electron capture dissociation (ECD), for determining the binding site of a small molecule ligand to a protein [8]. Earlier work by Zubarev and coworkers had suggested that weak, noncovalent intermolecular bonds could be preserved upon ECD [9]. We applied their initial findings to localize the binding site of a polyamine compound, spermine, to $13 \mathrm{kDa} \alpha$-synuclein [8]. Spermine had been demonstrated previously to enhance the propensity of $\alpha$-synuclein aggregation in Parkinson's disease. More commonly employed collisionally activated dissociation (CAD) for top-down MS readily dissociated the spermine ligand from the protein. However, using $\mathrm{ECD}$, the spermine ligand remains bound to specific $\mathrm{c}-/ \mathrm{z}$-product ions to localize spermine binding to the acidic C-terminal region of the protein. Thus, although the solution binding association for the $\alpha$-synuclein/ spermine complex is relatively weak $\left(k_{d} \sim 10^{-3} \mathrm{M}\right)$, ligand binding is retained in the gas phase and even upon ECD [8]. Moreover, these data suggest that many targeted aspects of the intermolecular associations formed initially in solution are preserved upon transition to the gas phase; the spermine binding sites determined by ESI-ECD-MS overlapped significantly with those measured by solution NMR.

In this report, we extend our initial studies by demonstrating that top-down MS with both ECD and $\mathrm{CAD}$ can be used to determine the ligand binding sites for specific protein-ligand complexes that are particularly stable in the gas-phase, such as the interaction between proteins and nucleotides. The stability of gasphase noncovalent complexes depends highly on the nature of the binding interactions $[8,10]$. For example, ribonuclease $S$ (RNase $S$ ) is a protein complex composed of the $11.5 \mathrm{kDa}$ S-protein and the $2.2 \mathrm{kDa}$ S-peptide that are noncovalently bound through hydrophobic interactions in solution with a $\mathrm{k}_{\mathrm{d}}$ of ca. $10^{-9} \mathrm{M}$. Maintaining such interactions in the gas phase is difficult because of the extreme instability of hydrophobic interactions in a waterless environment [11, 12]. However, through optimized instrumental parameters to minimize ion activation in the atmosphere/vacuum interface of the ESI source, the S-protein/S-peptide complex can be measured intact by ESI-MS $[10,13,14]$.

On the other end of the stability scale, the strength of electrostatic interactions is significantly enhanced in the absence of solvent $[2,10,15-19]$. The influence of solvent on electrostatic interactions and their role in noncovalent complexes has been described [11, 20,21]. With covalent-like strength, gas-phase electrostatic interactions are sustained even in CAD MS/MS experiments [10, 19, 22, 23]. For example, RNase A and cytidine $5^{\prime}$-triphosphate (CTP) form a noncovalent complex $\left(\mathrm{k}_{\mathrm{d}} \sim 10^{-6} \mathrm{M}\right)$ that involves electrostatic interactions. Woods et al. had previously reported on the enhanced gas-phase stability of phosphate anion inter- actions with basic amino acids found in a number of peptide systems $[16,17,19]$. Our group recently showed that CAD-MS/MS of the gas-phase RNase A-CTP complex results in fragmentation of the CTP ligand and formation of free CMP and RNase A firmly retaining a diphosphate group as the primary products [10]. The electrostatic interactions between the diphosphate group and RNase A are preserved. Such covalent-like strength of gas-phase electrostatic interactions provides us with potential opportunities to identify and characterize binding sites of protein-ligand complexes that involve similarly strong electrostatic interactions. Here, we show how the stability of electrostatic interactions can be exploited by top-down mass spectrometry to determine the adenosine 5 '-triphosphate (ATP) binding site of a protein kinase, adenylate kinase.

\section{Experimental}

\section{Materials}

Adenosine 5'-monophosphate (AMP), ATP, and adenylate kinase (AK; myokinase, from chicken muscle, product number M5520) were purchased from SigmaAldrich (St. Louis, MO, USA). All protein samples were desalted and concentrated with $10 \mathrm{mM}$ ammonium acetate buffer ( $\mathrm{pH}$ 6.6), using centrifugal filter devices (10 kDa MWCO, Amicon Ultra; Millipore Corp., Billerica, MA, USA). After desalting, ATP was added to a $5 \mu \mathrm{M}$ AK solution in ammonium acetate. The solution protein and ligand ratio was kept at 1:1.

\section{Mass Spectrometry Experiments}

A nanoESI source and $\mathrm{Au} / \mathrm{Pd}$ coated borosilicate glass capillaries (Proxeon Biosystems, Odense, Denmark), with flow rate around $50 \mathrm{~nL} / \mathrm{min}$, were coupled to a 7-Tesla LTQ-FT Ultra mass spectrometry (Thermo Fisher Scientific, San Jose, CA, USA) to acquire positive ionization mode ESI-MS spectra.

In the source region of the LTQ-FT Ultra, the capillary temperature was set to $210^{\circ} \mathrm{C}$, the capillary voltage was $+45 \mathrm{~V}$, and the tube lens was set to $+225 \mathrm{~V}$. The resolution of the Fourier-transform ion cyclotron resonance (FT-ICR) measurements was established to be 200,000 at 400 mass-to-charge ratio $(\mathrm{m} / \mathrm{z})$. The linear ion trap (LTQ) tuning was optimized to maximize the signal intensity of the ion at $\mathrm{m} / \mathrm{z} 2160$ for the 10+charged molecule of native AK. The target ion number for the LTQ and FT-ICR full MS scan was $3 \times 10^{4}$ and $1 \times 10^{5}$, respectively. For MS/MS experiments (e.g., precursor ion isolation), the target ion number for the LTQ and FT-ICR was $1 \times 10^{5}$. Three precursor ion isolation steps were carried out to optimize isolation of the targeted precursor ion and to minimize contributions from nearby ions. The initial isolation window was set to be centered with a slightly smaller $\mathrm{m} / \mathrm{z}$ than the targeted precursor ion and with a wider isolation width to reduce contamination from higher $\mathrm{m} / \mathrm{z}$ ions. 
The second isolation window was centered with a higher $m / z$ to reduce the lower $m / z$ contamination ions. The last isolation step was centered at the exact $m / z$ of the targeted precursor with a relative small isolation window. The isolation width was ca. $10-15 \mathrm{~m} / \mathrm{z}$ wide. Contaminations from higher $\mathrm{m} / \mathrm{z}$ ions were not avoided completely, but it was kept as low as possible with this procedure.

Further experimental parameters for top-down $\mathrm{MS}^{2}$ and $\mathrm{MS}^{3}$ are listed in Supplemental Table 1, which can be found in the electronic version of this article. Topdown MS/MS were accomplished with CAD or activated ion ECD (aiECD) in which ECD is coupled with infrared laser-heating of the product ions. Product ion yields are enhanced by IR laser-heating after ECD, consistent with hydrogen bond-retention upon ECD, but dissociation with further heating [24, 25]. Activated ion ECD was used effectively for our previous study on the $\alpha$-synuclein/spermine complex [8]. The 9+- and 10+-charged apo- and holo-forms of AK were analyzed with both CAD and aiECD. Further $\mathrm{MS}^{3}$ of AK-pp (AK bound to a diphosphate group) was carried out with CAD and aiECD as well. First, the AK-pp precursor ions were isolated in the LTQ after CAD-MS/MS of AK-ATP. For CAD of AK-pp, the isolated precursors were fragmented in the LTQ and then transferred to the FT-ICR for detection. For the aiECD experiment, precursors were first transferred to the FT-ICR cell and then dissociated into $\mathrm{c}-/ \mathrm{z}^{-}$-product ions. Product ion mass measurement accuracy was generally better than $7 \mathrm{ppm}$.

\section{Results and Discussion}

Kinases are enzymes that transfer a phosphoryl group from ATP to the hydroxyl groups of proteins or mononucleotides. Phosphorylated proteins and nucleotides are involved in many biological processes, such as signaling and maintaining intracellular nucleotide pools. The interaction between adenylate kinase and ATP serves as a good model system for our top-down MS/MS methodology because the ATP-binding sites of AK have long been investigated by NMR [26, 27], X-ray crystallography [28-30], and photoactive labeling [31].

AK (ATP:AMP phosphotransferase, EC 2.7.4.3) has a molecular weight of $21-23 \mathrm{kDa}$. There are at least five major families of AK: AK1 is from the cytosol of mammalian or vertebrate muscles, AK2 is located in the mammalian mitochondrial intermembrane, AK3 is from the mammalian mitochondrial matrix, $\mathrm{AKe}$ is found in Escherichia coli, AKy is generated by Saccharomyces cerevisiae [27], and a recently reported AK6 minor family in cell nucleus [32]. The ATP binding sites of AK have been studied in many different AK families, including chicken, human, porcine, rabbit AK1, and AKe. AK1 from rabbit (KAD1_Rabit), porcine (KAD1_Pig), and human (KAD1_human) have 93\%, $92 \%$, and $92 \%$ sequence homology, respectively, to chicken AK1 (AK1c). AKe (KAD_Ecoli) shares 55\% homology in sequence with AK1c. In this study, AK1c was used and the amino acids are numbered according to the AK1c sequence.

\section{CAD-MS/MS of AK, AK-AMP, and AK-ATP}

ESI mass spectra for nucleotide-bound AK have been reported previously [33-36]. Our ESI-FT-ICR-MS and MS/MS spectra of AK (measured average MW 21,593.8 \pm 0.1 Da, expected 21,593.9 Da; Figure 1a), the AK-AMP complex (measured average MW 21,940.9 $\pm 0.1 \mathrm{Da}$, expected 21,941.1; Supplemental Figure 1a), and the AK-ATP complex (measured average MW 22,101.2 \pm $0.1 \mathrm{Da}$, expected 22,101.1 Da; Figure 1b) were acquired with the LTQ-FT mass spectrometer.

Abundant 1:1 AK-AMP and AK-ATP noncovalent complexes of relative low charge, $9+$ and $10+$, were measured, consistent with most noncovalent complexes measured from near neutral $\mathrm{pH}$ solutions with ESI-MS. CAD of the $9+$ and $10+$ AK-AMP complexes yielded primarily the loss of a neutral-charged AMP ligand and the apo-AK protein (Supplemental Figure 1b). This behavior is similar to our previous data for CMP-bound RNase A [10]. However, also consistent with our previously reported CAD MS/MS spectra measured for the RNase A-CTP complex [10], CAD of AK-ATP released AMP and generated the AK-pp complex, i.e., AK bound to a diphosphate group adduct $\left(\mathrm{H}_{3} \mathrm{P}_{2} \mathrm{O}_{6}\right)$, as well as $b$-/y-product ions from the polypeptide backbone (Figure 2 for the $10+$ precursor, and Supplemental Figure 2 for the $9+$ precursor). Figure 3 depicts the primary dissociation pathways of the AK-ATP fragmentation. Also measured in the primary CAD mass spectra from the AK-ATP complex were b-/y-products retaining intact ATP and a diphosphate group. MS/MS of AK-pp from $\mathrm{MS}^{3}$ experiments provide complementary information about the putative ATP binding sites to AK. They also provide information about a potential conformational change of AK upon ATP binding (vide infra).

Dissociation of the amide bond C-terminal to an Asp residue was especially prevalent in the top-down CAD experiments. Other laboratories have reported an unusually elevated proportion of dissociation products C-terminal to Asp residues [37-39]. CAD-MS/MS of the 9+ apo-AK (Supplemental Table 2) produced 37 fragments covering $19 \%$ of the AK polypeptide sequence. Within these 37 fragments, there are 8 fragmentation sites that are particularly abundant, and products from these sites were measured with more than one charge state. Six out of these eight abundant fragmentation sites are from cleavage of the amide bond C-terminal to an Asp. In CAD-MS/MS of the 10+ apo-AK (Figure 2), 12 abundant products are from the C-terminal bond to an Asp residue. Both $9+$ and $10+$ AK-ATP show four abundant fragments in their MS/MS spectra; most of these sites originate from the C-terminal bond to an Asp.

A large proportion, greater than $90 \%$ in some examples, of the total signal in the CAD mass spectra of holo-AK were found in the (AK-pp) ${ }^{n+}$ product. Lower 


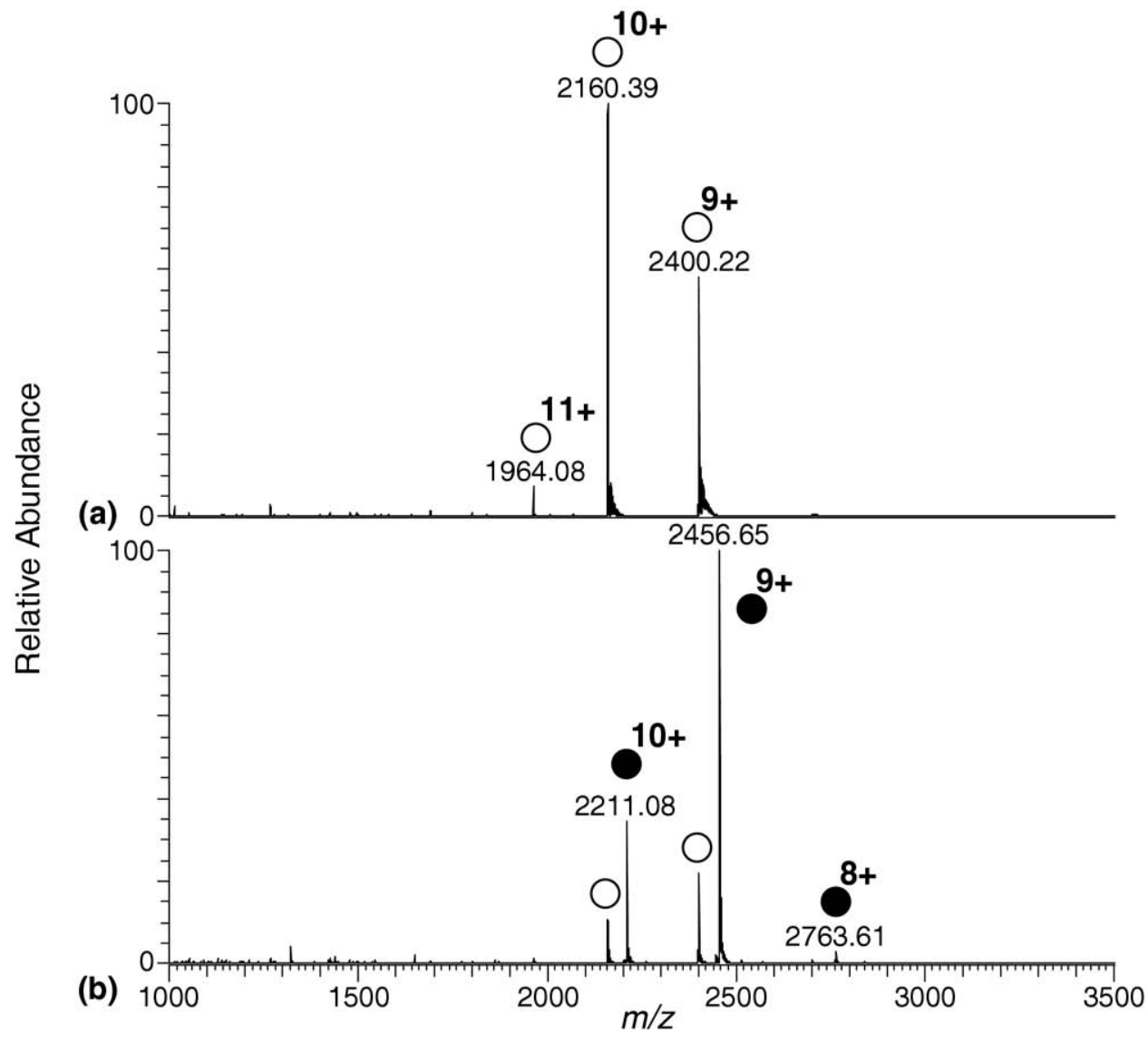

Figure 1. ESI mass spectra of (a) AK and (b) AK in the presence of ATP. The peaks marked with open circles represent multiply charged molecules of the apo-protein, and those marked with filled circles represent the 1:1 AK-ATP complex.

abundant $\mathrm{b} / \mathrm{y}$ products that also retain the diphosphate group, e.g., $\mathrm{y}_{100}-\mathrm{pp}$ and $\mathrm{b}_{180}-\mathrm{pp}$, were observed that may or may not have resulted from secondary fragmentation of the AK-pp species (Supplemental Figure 2 and Figure 2).

CAD-MS ${ }^{3}$ of AK-pp (Figure 4) also generated data to identify the ATP binding site. Loss of a phosphate group from AK-pp to generate the AK-p species was the most abundant product. However, other $b-/ y-$ products with mono- and di-phosphate bound were measured as well. More importantly, the $\mathrm{MS}^{3}$ data confirmed the binding site identified from CADMS/MS of AK-ATP. Holo-fragments, including those retaining either intact ATP or the diphosphate group, were gathered to plot a map on which an ATP binding site is found in between amino acid 121-140 (Figure 5). Results from $\mathrm{MS}^{3}$ of AK-pp confirmed that the diphosphate group bound to fragments at the same site as the ATP. The newly observed $\mathrm{y}_{53}-\mathrm{pp}$ ion from $\mathrm{MS}^{3}$ of AK-pp pinpoints another weaker binding site of the nucleotide between amino acid 141-180 (Figure 5).

\section{ECD-MS/MS of AK-ATP}

Although ECD efficiency can be very high to promote top-down MS studies, ECD by itself did not provide significantly more information about the ligand binding site for the AK-ATP complex. Precursor ion charge states affect aiECD experiments significantly because electron capture cross sections increase quadratically with charge [40]. For aiECD of the 9+-charged holo-AK, the coverage and number of fragments were at least $50 \%$ less than those in aiECD of the $10+$ precursor (Supplemental Table 2). No holo-fragments were observed from aiECD of the $9+$ molecule. Although aiECD has a much lower fragmentation intensity compared with $C A D$, it still provided additional valuable data that verified the ATP binding sites determined by CAD-MS/MS and $\mathrm{MS}^{3}$ (Figure 5).

Product ions from aiECD-MS/MS originated mainly from the $\mathrm{N}$-terminal region of the protein, whereas those from CAD were more evenly distributed along protein sequence. The aiECD-MS/MS of $10+$ and $9+$ ions generated more c-product ions than $\mathrm{z}$-ions; the ratio of c- to z-ions from AK-ATP and AK-pp precursors was observed to be 8.5 and 5 , respectively. In consideration of all MS/MS fragments from both charge states of apo-AK, the sequence coverage of CAD and aiECD was found to be $32 \%$ and $41 \%$, respectively (Supplemental Table 2). Combined data of CAD and aiECD gave nearly $61 \%$ sequence coverage of apo-AK, which is much higher than each individual top-down 


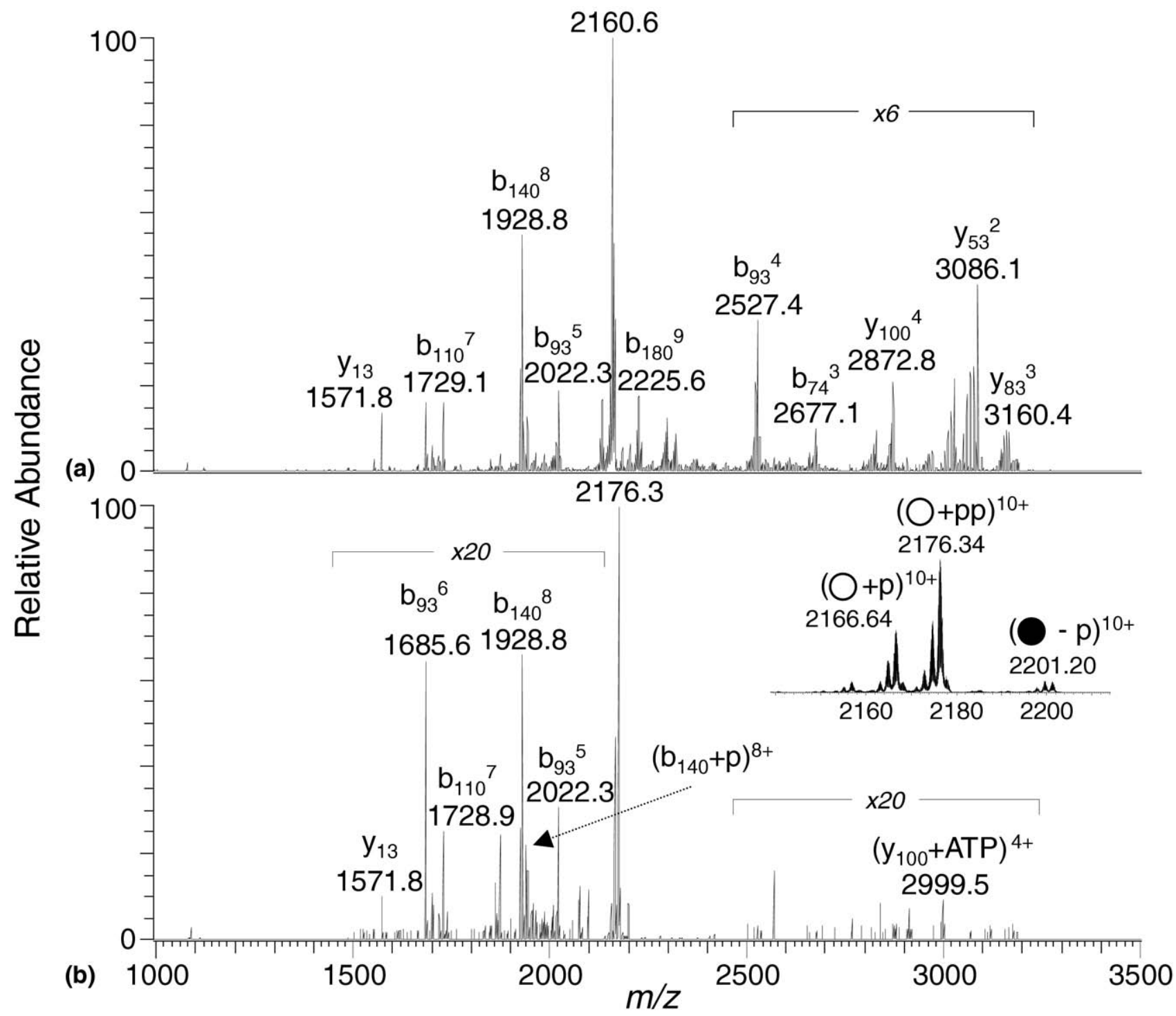

Figure 2. ESI CAD-tandem mass spectra of the 10+-charged (a) AK and (b) AK-ATP 1:1 complex. Product ions of the intact apo-protein are marked by open circles, and those of the holo-protein are marked with the filled circle. Retention of one phosphate group by the product ion is represented by " $\mathrm{p}$ ", and retention of two phosphates is labeled with "pp." The loss or gain of a phosphate group is labeled with " $-\mathrm{p}$ " or " $+\mathrm{p}$ ", respectively.

method alone. Such phenomena were also observed from MS/MS of AK-ATP, and MS ${ }^{3}$ of AK-pp.

In summary, distribution maps of apo and holo fragments were generated from combined $\mathrm{CAD}$ and aiECD data from both charge states of apo-AK, AKATP, and AK-pp (Figure 5). After comparing the location of holo fragments and apo fragments on the CAD-MS/MS and $\mathrm{MS}^{3}$ map, one primary ATP binding site is located in between amino acids 121-140, and a secondary binding site is observed between residues 141-180. ECD data confirms that there is at least one binding site between residues 94-150. Thus, ECD in this case did not provide more detailed information about defining the ligand binding sites, but they directly corroborate binding regions that were identified by CAD.

\section{Dissociation Efficiency of $A K$ and $A K-A T P$}

The CAD efficiency (i.e., generation of detectable product ions) was greatly affected by the presence of ATP ligand binding. Overall sequence coverage of AK from CAD of the $9+$ and $10+$ AK-ATP complex (32\%) was significantly lower than that from CAD of the apo-AK molecule (14\%) (Supplemental Table 2), even though a slightly higher activation energy was used for CAD of the holo-AK complex. This sequence coverage behavior may be partially explained by the observation that a majority of the CAD product ion signal was found in the (AK-pp $)^{n+}$ product rather than distributed among products resulting from amide backbone bond cleavages. 


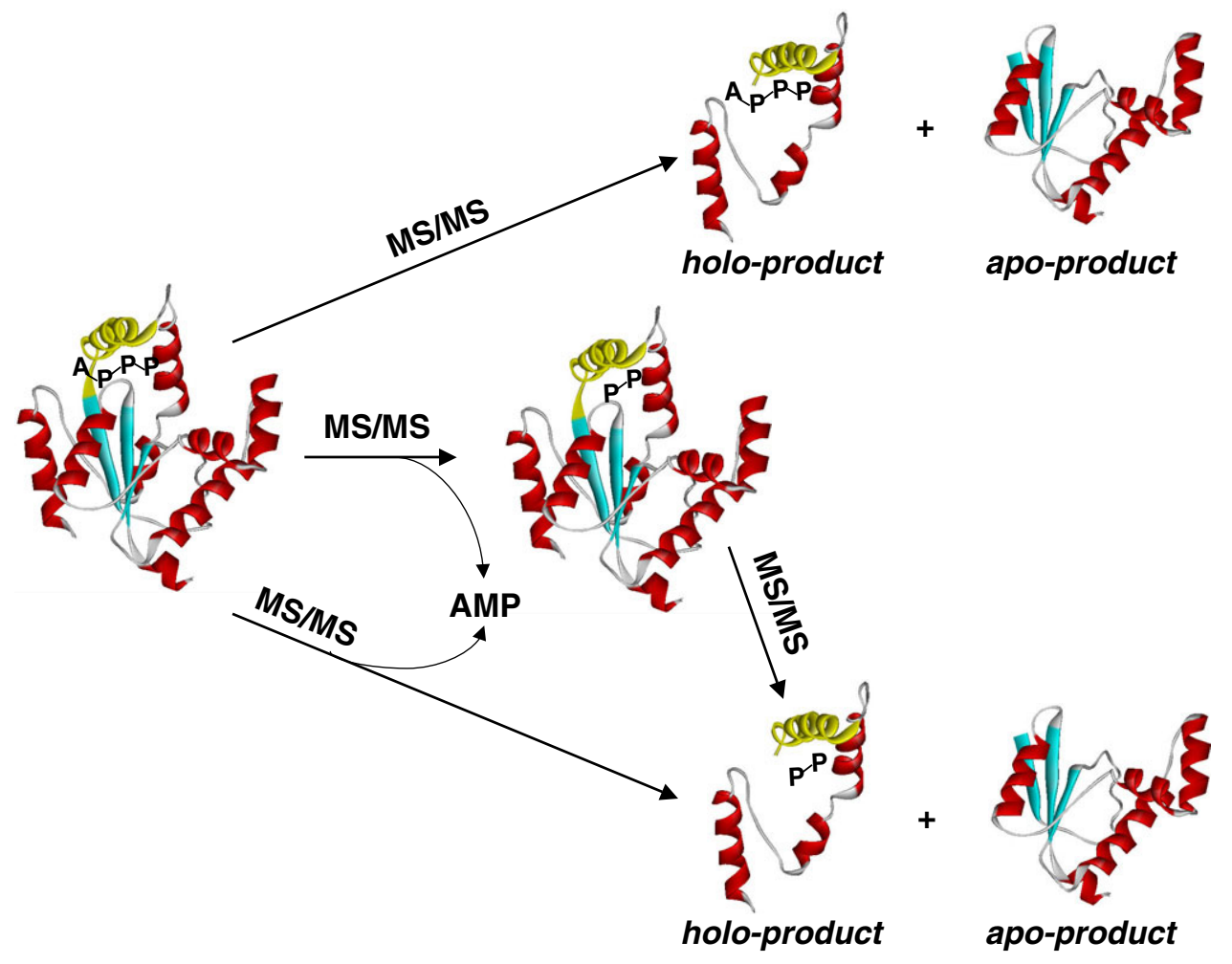

Figure 3. Dissociation pathways for the AK-ATP complex observed by top-down MS.

However, aiECD efficiency leading to formation of sequence informative product ions was not affected by the presence of ATP ligand binding. Sequence coverage from aiECD of apo-AK and holo-AK were similar, 41\% and $39 \%$, respectively. Moreover, the total number of unique product ions generated by aiECD was similar for both species: 79 for apo-AK and 75 for holo-AK. It is not clear if a ligand-induced solution-phase conformational change suggested for ATP-binding by AK [27, 41, 42] is affecting the dissociation behavior of the gas-phase AK-ATP complex, i.e., CAD efficiencies are significantly reduced for the ATP-bound protein, following the conversion from an open to closed conformation with ATP binding [41, 42]. Based on previous work by others,

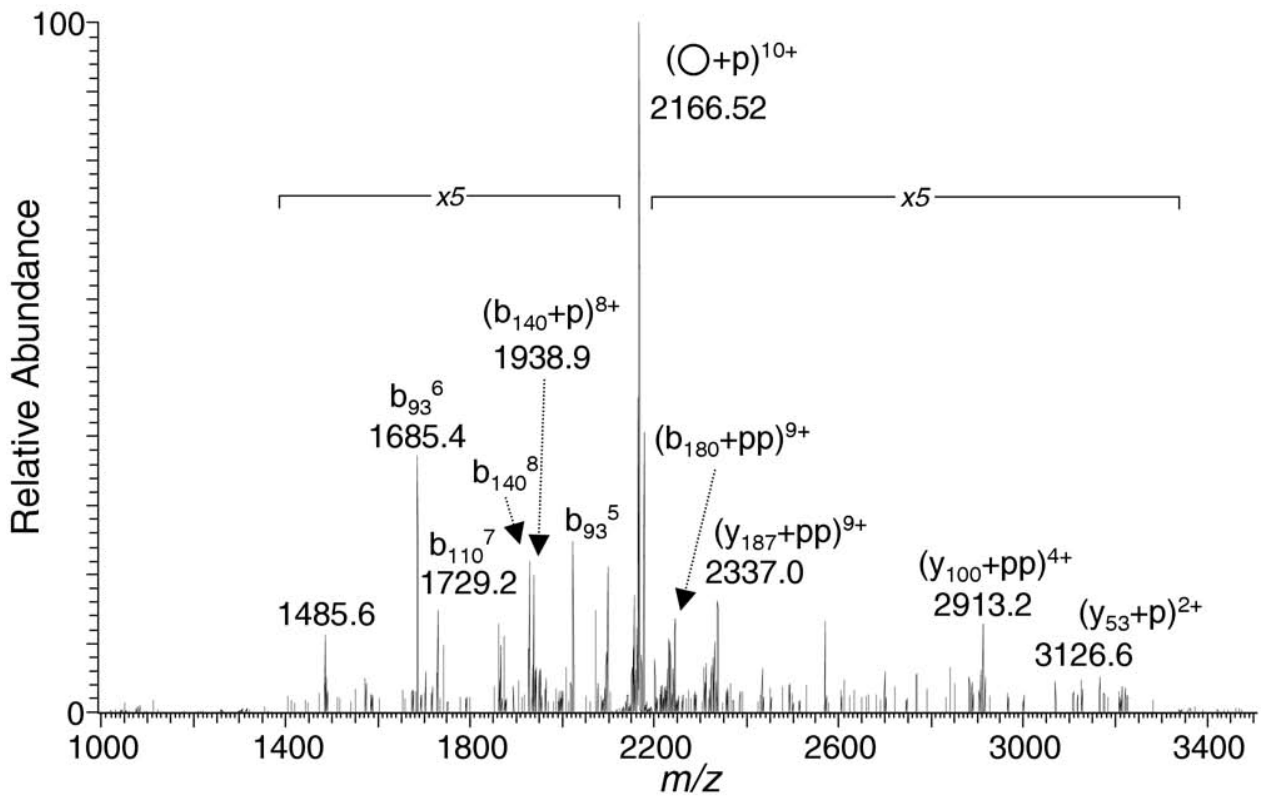

Figure 4. CAD-MS/MS spectrum $\left(\mathrm{MS}^{3}\right)$ of the $10+\mathrm{AK}$-pp product ion $(\mathrm{m} / \mathrm{z} 2176$; see Figure 2 legend for description of labels). 


\title{
MS/MS Apo-AK
}

\section{$N$-terminal ions}

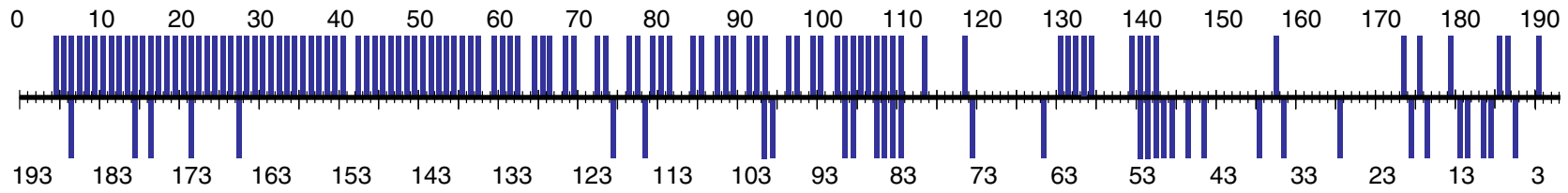

(a)

\section{MS/MS AK-ATP}

C-terminal ions

\section{$N$-terminal ions}

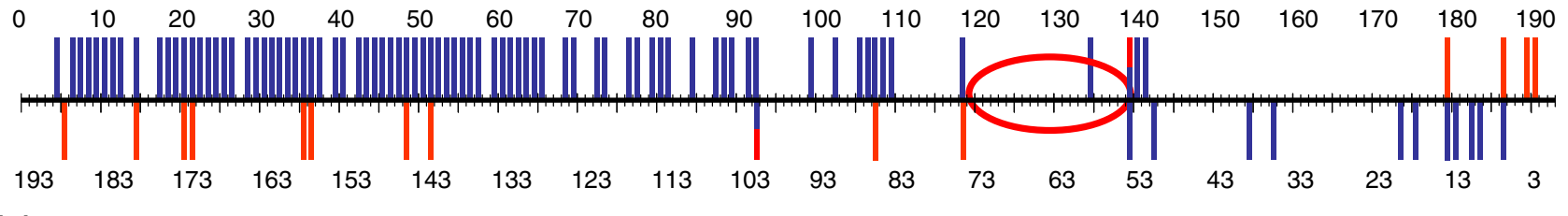

(b)

\section{MS/MS (MS $\left.{ }^{3}\right)$ AK-pp}

\section{$N$-terminal ions}

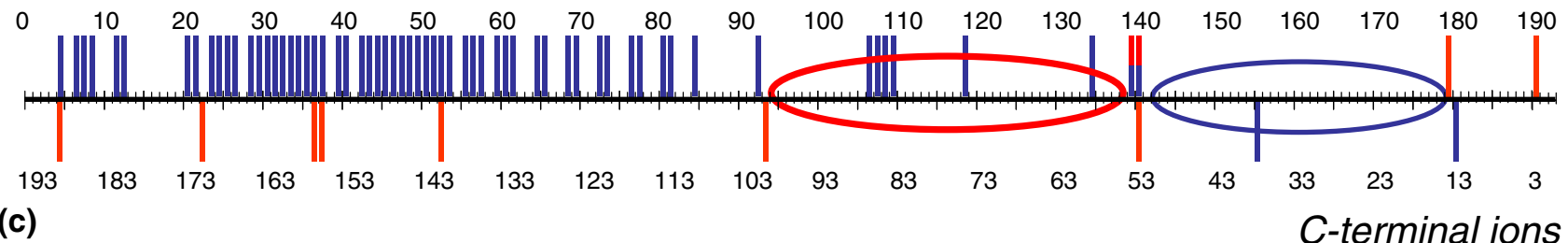

\begin{abstract}
Figure 5. Combined product ion distributions for the 9+ and 10+ (a) apo-AK, (b) AK-ATP, and (c) AK-pp measured by CAD and ECD. The b/c product ions are represented by the bars above the primary sequence line, and the $\mathrm{y} / \mathrm{z}$ products are represented by the bars below the sequence line. Product ions that retain a phosphate (p), a diphosphate (pp), or ATP are represented by the red bars (i.e., holo-products). Bars with two colors indicate overlapping apo- and holo-fragments from either CAD or ECD. The ovals span the putative ATP-binding regions.
\end{abstract}

including McLafferty [43], O'Connor [44], and Williams [45], it should be possible to probe differences in protein conformation by ECD. However, from our data for AK and the AK-ATP complex, aiECD efficiencies appear to depend more on charge state than conformation; sequence coverage and the number of products increases from $31 \%$ and $16 \%$ for the $9+$ apo-AK, respectively, to $79 \%$ and $41 \%$ for the 10+ charge state. (Similar ECD data were measured for the holo-AK complex.)

\section{ATP-Binding Sites Derived from Solution Phase and Mass Spectrometry Data}

In solution, AK contains two adjacent binding sites with different binding affinity for ATP and AMP [26, 46]. During the transformation of the phosphoryl group from ATP to AMP, AK must protect its catalytic center to avoid transferring the phosphoryl group to free water. Many studies using a variety of methodologies since the 1970s have been initiated to determine the solution phase site of ATP-binding to chicken AK, and many more have been reported for AKs from other organisms.
From the NMR data of rabbit muscle AK [26], the triphosphate portion of ATP is bound to K21, L27, and Q24. Similar conclusions were reached from analysis of chicken $\mathrm{AK}$, as the side chain of K21 forms hydrogen bonds with the second and third phosphates of ATP [47]. Thus, apparently this "essential" lysine plays several important structural and functional roles: it stabilizes the phosphate-binding loop (P-loop) and it orients the triphosphate of ATP to its proper conformation. X-ray crystallographic studies also strongly suggested the involvement of several highly conserved arginines, including R132, R138, R149, R44, R97, and the Gly-loop (residues 18-23) [27-29, 48].

In addition, R138 cannot be replaced by a lysine residue, indicating its essential role for catalysis [49]. Photoaffinity-labeling with 8-azidoadenine analogs suggested three possible bindings sites located between residues K27-L43, T124-E134, and T152-K165 [31]. Analysis of $E$. coli AK crystal structures with AMP, ADP, and $\mathrm{p}^{1}$, $\mathrm{p}^{5}$-di(adenosine $\left.5^{\prime}\right)$-pentaphosphate $\left(\mathrm{Ap}_{5} \mathrm{~A}\right)$ also assign R44, R97, R132, R149 as key residues for domain closure and substrate binding [30]. Data from human AK1 bound to $\mathrm{Ap}_{5} \mathrm{~A}$ shows similar interactions with the 
phosphate groups of the substrate [50]; human AK1 and chicken AK1 show $83 \%$ sequence identity. From most of the high-resolution structural data, apparently the Glyloop (residues 18-23) interacts with the $\alpha$-phosphate and $\beta$-phosphate, and R132 and R138 interact with the $\gamma$-phosphate of ATP $[29,30,41]$.

Top-down tandem mass spectrometry of AK-ATP identifies amino acids 121-140, also known as the "lidloop," to be the primary binding site of ATP to AK1c in the gas phase. An additional minor site was found also at residues 141-180. Our gas-phase data support the conserved arginines' responsibility for binding ATP. The Gly-loop near the N-terminus and part of the solution phase ATP-binding pocket is not implicated by the mass spectrometry data. However, this may be a result of the gas-phase process involved in the MS/MS event. CAD of AK-ATP releases free AMP (containing the $\alpha$-phosphate) to form the AK-pp species. If the primary interactions involved in the solution phase structure are retained in the gas-phase complex, residues R132 and R138 would be responsible for the retention of the $\gamma$-phosphate group in the AK-pp complex, and the interaction between the Gly-loop residues (primarily K21) and the $\beta$-phosphate may be too weak in the gas phase to survive the CAD events.

\section{Conclusions}

Many examples have been reported in which ESI was used to deliver a noncovalent protein complex from solution into the gas phase and was measured intact by mass spectrometry [2,51-54]. However, these studies generally do not address the question whether the structure of the gas-phase complex is similar to its initial solution phase structure. Because the phase transition from solution to the gas phase is not a subtle effect, it would not be surprising to find some perturbation in the structure of a protein complex upon removal of solvent. In fact, studies suggest that large protein complexes undergo a shrinking process as a result of solvent loss [1]. Therefore, the solution to Breuker and McLafferty's question, "For how long, under what conditions, and to what extent, can solution structure be retained without solvent?" is not entirely obvious from reports to date [55].

Our results from the top-down MS analysis of the gas-phase ATP-bound AK complex are generally consistent with previous data measured by NMR and X-ray crystallography. The ATP ligand and the AK protein are in equilibrium in solution, with a dissociation constant in the micromolar range [34, 35, 56]. Upon solvent removal to transition into the gas phase, protein conformation could be rapidly converting into "new, non-native structures" [55]. Even if the protein's conformation is undergoing rapid remolding into a new structure, for adenylate kinase, however, the ATP ligand appears to be fixed to a position dictated by its solution phase structure. The enhanced gas-phase electrostatic interactions allow the position of the ligand within the protein to be locked in place and allow further probing by tandem MS.

Thus, despite the fact that the solution threedimensional structure of a protein complex may not be completely retained in vacuo, top-down MS may be a novel, complementary method to higher resolution structural methods for determining the sites of ligand binding. We predict that protein-nucleotide complexes will be especially stable to such MS probing, and that the method may be useful for determining the sites of ATP-binding to protein kinases.

\section{Acknowledgments}

J.A.L. acknowledges support from the National Institutes of Health (RR 20004) and the NIH/NCRR High-End Instrumentation Program (S10 RR023045).

\section{Appendix A Supplementary Material}

Supplementary material associated with this article may be found in the online version at doi:10.1016/ j.jasms.2010.01.002.

\section{References}

1. Kaddis, C. S.; Lomeli, S. H.; Yin, S.; Berhane, B.; Apostol, M. I.; Kickhoefer, V. A.; Rome, L. H.; Loo, J. A. Sizing Large Proteins and Protein Complexes by Electrospray Ionization Mass Spectrometry and Ion Mobility. J. Am. Soc. Mass Spectrom. 2007, 18, 1206-1216.

2. Loo, J. A. Studying Noncovalent Protein Complexes by Electrospray Ionization Mass Spectrometry. Mass Spectrom. Rev. 1997, 16, 1-23.

3. Zhang, S.; Van Pelt, C. K.; Wilson, D. B. Quantitative Determination of Noncovalent Binding Interactions Using Automated Nanoelectrospray Mass Spectrometry. Anal. Chem. 2003, 75, 3010-3018.

4. Kelleher, N. L. Top-Down Proteomics. Anal. Chem. 2004, 76, 197A-203A

5. Ayaz-Guner, S.; Zhang, J.; Li, L.; Walker, J. W.; Ge, Y. In Vivo Phosphorylation Site Mapping in Mouse Cardiac Troponin I by High Resolution Top-Down Electron Capture Dissociation Mass Spectrometry: Ser22/23 are the Only Sites Basally Phosphorylated. Biochemistry 2009, 48, 8161-8170.

6. Pan, J.; Han, J.; Borchers, C. H.; Konermann, L. Hydrogen/Deuterium Exchange Mass Spectrometry with Top-Down Electron Capture Dissociation for Characterizing Structural Transitions of a $17 \mathrm{kDa}$ Protein. J. Am. Chem. Soc. 2009, 131, 12801-12808.

7. Loo, J. A. Probing Protein-Metal Ion Interactions by Electrospray Ionization Mass Spectrometry: Enolase and Nucleocapsid Protein. Int. J. Mass Spectrom. 2001, 204, 113-123.

8. Xie, Y.; Zhang, J.; Yin, S.; Loo, J. A. Top-Down ESI-ECD-FT-ICR Mass Spectrometry Localizes Noncovalent Protein-Ligand Binding Sites. J. Am. Chem. Soc. 2006, 128, 14432-14433.

9. Haselmann, K. F.; Jorgensen, T. J. D.; Budnik, B. A.; Jensen, F.; Zubarev, R. A. Electron Capture Dissociation of Weakly Bound Polypeptide Polycationic Complexes. Rapid Commun. Mass Spectrom. 2002, 16, 2260 2265.

10. Yin, S.; Xie, Y.; Loo, J. A. Mass Spectrometry of Protein-Ligand Complexes: Enhanced Gas-Phase Stability of Ribonuclease-Nucleotide Complexes. J. Am. Soc. Mass Spectrom. 2008, 19, 1199-1208.

11. Daniel, J. M.; Friess, S. D.; Rajagopalan, S.; Wendt, S.; Zenobi, R. Quantitative Determination of Noncovalent Binding Interactions Using Soft Ionization Mass Spectrometry. Int. I. Mass Spectrom. 2002, 216, 1-27.

12. Liu, L.; Bagal, D.; Kitova, E. N.; Schnier, P. D.; Klassen, J. S. Hydrophobic Protein-Ligand Interactions Preserved in the Gas Phase. J. Am. Chem. Soc. 2009, 131, 15980-15981.

13. Goodlett, D. R.; Ogorzalek Loo, R. R.; Loo, J. A.; Wahl, J. H.; Udseth H. R.; Smith, R. D. A Study of the Thermal Denaturation of Ribonuclease $\mathrm{S}$ by Electrospray Ionization Mass Spectrometry. J. Am. Soc. Mass Spectrom. 1994, 5, 614-622.

14. Ogorzalek Loo, R. R.; Goodlett, D. R.; Smith, R. D.; Loo, J. A. Observation of a Noncovalent Ribonuclease S-Protein/S-Peptide Complex by Electrospray Ionization Mass Spectrometry. J. Am. Chem. Soc. 1993, 115, 4391-4392.

15. Alves, S.; Woods, A.; Tabet, J. C. Charge State Effect on the Zwitterion Influence on Stability of Noncovalent Interaction of Single-Stranded DNA with Peptides. J. Mass Spectrom. 2007, 42, 1613-1622. 
16. Jackson, S. N.; Wang, H. Y. J.; Woods, A. S. Study of the Fragmentation Patterns of the Phosphate-Arginine Noncovalent Bond. I. Proteome. Res. 2005, 4, 2360-2363.

17. Jackson, S. N.; Wang, H. Y. J.; Yergey, A.; Woods, A. S. Phosphate Stabilization of Intermolecular Interactions. J. Proteome Res. 2006, 5, 122-126.

18. Woods, A. S. The Mighty Arginine, the Stable Quaternary Amines, the Powerful Aromatics, and the Aggressive Phosphate: Their Role in the Noncovalent Minuet. J. Proteome Res. 2004, 3, 478-484.

19. Woods, A. S.; Ferre, S. Amazing Stability of the Arginine-Phosphate Electrostatic Interaction. J. Proteome Res. 2005, 4, 1397-1402.

20. Kitova, E. N.; Seo, M.; Roy, P.-N.; Klassen, J. S. Elucidating the Intermolecular Interactions within a Desolvated Protein-Ligand Complex. An Experimental and Computational Study. J. Am. Chem. Soc. 2008, 130, 1214-1226.

21. Strittmatter, E. F.; Williams, E. R. The Role of Proton Affinity, Acidity, and Electrostatics on the Stability of Neutral Versus Ion-Pair Forms of Molecular Dimers. Int. J. Mass Spectrom. 2001, 212, 287-300.

22. Jackson, S. N.; Dutta, S.; Woods, A. S. The Use of ECD/ETD to Identify the Site of Electrostatic Interaction in Noncovalent Complexes. J. Am. Soc. Mass Spectrom. 2009, 20, 176-179.

23. Sundqvist, G.; Benkestock, K.; Roeraade, J. Investigation of Multiple Binding Sites on Ribonuclease A Using Nano-Electrospray Ionization Mass Spectrometry. Rapid Commun. Mass Spectrom. 2005, 19, 1011-1016.

24. Breuker, K.; Oh, H.; Lin, C.; Carpenter, B. K.; McLafferty, F. W. Nonergodic and Conformational Control of the Electron Capture Dissociation of Protein Cations. Proc. Natl. Acad. Sci. U.S.A. 2004, 101, 14011-14016.

25. Oh, H.; Breuker, K.; Sze, S. K.; Ge, Y.; Carpenter, B. K.; McLafferty, F. W. Secondary and Tertiary Structures of Gaseous Protein Ions Characterized by Electron Capture Dissociation Mass Spectrometry and Photofragment Spectroscopy. Proc. Natl. Acad. Sci. U.S.A. 2002, 99, 1586315868

26. Fry, D. C.; Kuby, S. A.; Mildvan, A. S. NMR-Studies of the MgATP Binding-Site of Adenylate Kinase and of a 45-Residue Peptide Fragment of the Enzyme. Biochemistry 1985, 24, 4680-4694.

27. Tsai, M. D.; Yan, H. Mechanism of Adenylate Kinase: Site-Directed Mutagenesis Versus X-Ray and NMR. Biochemistry 1991, 30, 6806-6818.

28. Pai, E. F.; Sachsenheimer, W.; Schirmer, R. H.; Schulz, G. E. Substrate Positions and Induced-Fit in Crystalline Adenylate Kinase. J. Mol. Biol. 1977, 114, 37-45.

29. Schulz, G. E. Induced-Fit Movements in Adenylate Kinases. Faraday Discuss. 1992, 85-93.

30. Berry, M. B.; Bae, E. Y.; Bilderback, T. R.; Glaser, M.; Phillips, G. N. Crystal Structure of ADP/AMP Complex of Escherichia coli Adenylate Kinase. Proteins Struct. Funct. Bioinform. 2006, 62, 555-556.

31. Salvucci, M. E.; Chavan, A. J.; Haley, B. E. Identification of Peptides from the Adenine Binding Domains of ATP and AMP in Adenylate Kinase-Isolation of Photoaffinity-Labeled Peptides by Metal Chelate Chromatography. Biochemistry 1992, 31, 4479-4487.

32. Ren, H.; Wang, L. Y.; Bennett, M.; Liang, Y. H.; Zheng, X. F.; Lu, F.; Li, L. F.; Nan, J.; Luo, M.; Eriksson, S.; Zhang, C. M.; Su, X. D. The Crystal Structure of Human Adenylate Kinase 6: An Adenylate Kinase Localized to the Cell Nucleus. Proc. Natl. Acad. Sci. U.S.A. 2005, 102, 303-308.

33. Briand, G.; Perrier, V.; Kouach, M.; Takahashi, M.; Gilles, A.-M.; Barzu, $\mathrm{O}$. Characterization of Metal and Nucleotide Liganded Forms of Adenylate Kinase by Electrospray Ionization Mass Spectrometry. Arch. Biochem. Biophys. 1997, 339, 291-297.

34. Daniel, J. M.; McCombie, G.; Wendt, S.; Zenobi, R. Mass Spectrometric Determination of Association Constants of Adenylate Kinase with Two Noncovalent Inhibitors. J. Am. Soc. Mass Spectrom. 2003, 14, 442-448.

35. Jecklin, M. C.; Touboul, D.; Bovet, C.; Wortmann, A.; Zenobi, R. Which Electrospray-Based Ionization Method Best Reflects Protein-Ligand Interactions Found in Solution? A Comparison of ESI, NanoESI, and ESSI for the Determination of Dissociation Constants with Mass Spectrometry. J. Am. Soc. Mass Spectrom. 2008, 19, 332-343.
36. Lomeli, S. H.; Peng, I. X.; Yin, S.; Ogorzalek Loo, R. R.; Loo, J. A. New Reagents for Increasing ESI Multiple Charging of Proteins and Protein Complexes. J. Am Soc. Mass Spectrom. 2010, 21, 127-131.

37. Mekecha, T. T.; Amunugama, R.; McLuckey, S. A. Ion Trap CollisionInduced Dissociation of Human Hemoglobin $\alpha$-Chain Cations. J. Am. Soc. Mass Spectrom. 2006, 17, 923-931.

38. Qin, J.; Chait, B. T. Preferential Fragmentation of Protonated Gas-Phase Peptide Ions Adjacent to Acidic Amino Acid Residues. J. Am. Chem. Soc 1995, 117, 5411-5412.

39. Tsaprailis, G.; Somogyi, Á.; Nikolaev, E. N.; Wysocki,V. H. Refining the Model for Selective Cleavage at Acidic Residues in Arginine Containing Protonated Peptides. Int. J. Mass Spectrom. 2000, 195, 467-479.

40. Zubarev, R. A.; Horn, D. M.; Fridriksson, E. K.; Kelleher, N. L.; Kruger N. A.; Lewis, M. A.; Carpenter, B. K.; McLafferty, F. W. Electron Capture Dissociation for Structural Characterization of Multiply Charged Protein Cations. Anal. Chem. 2000, 72, 563-573.

41. Müller, C. W.; Schlauderer, G. J.; Reinstein, J.; Schulz, G. E. Adenylate Kinase Motions During Catalysis: An Energetic Counterweight Balancing Substrate Binding. Structure 1996, 4, 147-156.

42. Rundqvist, L.; Åden, J.; Sparrman, T.; Wallgren, M.; Olsson, U.; WolfWatz, M. Non-cooperative Folding of Subdomains in Adenylate Kinase. Biochemistry 2009, 48, 1911-1927.

43. Breuker, K.; McLafferty, F. W. The Thermal Unfolding of Native Cytochrome $c$ in the Transition from Solution to Gas Phase Probed by Native Electron Capture Dissociation. Angew. Chem. Int. Ed. 2005, 44, 4911-4914.

44. Lin, C.; Cournoyer, J. J.; O'Connor, P. B. Probing the Gas-Phase Folding Kinetics of Peptide Ions by IR Activated DR-ECD. I. Am. Soc. Mass Spectrom. 2008, 19, 780-789.

45. Robinson, E. W.; Leib, R. D.; Williams, E. R. The Role of Conformation on Electron Capture Dissociation of Ubiquitin. J. Am. Soc. Mass Spectrom. 2006, 17, 1469-1479.

46. Noda, L. Adenosine Triphosphate-Adenosine Monophosphate Transphosphorylase. 3. Kinetic Studies. J. Biol. Chem. 1958, 232, 237-250.

47. Byeon, I.-J. L.; Shi, Z.; Tsai, M.-D. Mechanism of Adenylate Kinase. The Essential Lysine Helps to Orient the Phosphates and the Active Site Residues to Proper Conformations. Biochemistry 1995, 34, 3172-3182.

48. Schulz, G. E.; Müller, C. W.; Diederichs, K. Induced-Fit Movements in Adenylate Kinases. J. Mol. Biol. 1990, 213, 627.

49. Yan, H.; Shi, Z.; Tsai, M. D. Mechanism of Adenylate Kinase. Structural and Functional Demonstration of Arginine-138 as a Key Catalytic Residue That Cannot Be Replaced by Lysine. Biochemistry 1990, 29 , 6385-6392.

50. Bunkoczi, G.; Filippakopoulos, P.; Jansson, A.; Longman, E.; Delft, F. V.; Edwards, A.; Arrowsmith, C.; Sundstrom, M.; Knapp, S. Structure of Adenylate Kinase 1 In Complex With $\mathrm{P}^{1}, \mathrm{P}^{4}$-Di(Adenosine)Tetraphosphate (RCSB Protein Data Bank; PDB ID 2C95; www.pdb.org).

51. Heck, A. J. R.; van den Heuvel, R. H. H. Investigation of Intact Protein Complexes by Mass Spectrometry. Mass Spectrom. Rev. 2004, 23, 368 389.

52. Loo, J. A. Electrospray Ionization Mass Spectrometry: A Technology for Studying Noncovalent Macromolecular Complexes. Int. J. Mass Spectrom. 2000, 200, 175-186.

53. Lomeli, S. H.; Yin, S.; Ogorzalek Loo, R. R.; Loo, J. A. Increasing Charge While Preserving Noncovalent Protein Complexes for ESI-MS. J. Am. Soc. Mass Spectrom. 2009, 20, 593-596.

54. Barrera, N. P.; Bartolo, N. D.; Booth, P. J.; Robinson, C. V. Micelles Protect Membrane Complexes from Solution to Vacuum. Science 2008, 321, 243-246

55. Breuker, K.; McLafferty, F. W. Stepwise Evolution of Protein Native Structure with Electrospray into the Gas Phase, $10^{-12}$ to $10^{2}$ s. Proc. Natl. Acad. Sci. U.S.A. 2008, 105, 18145-18152.

56. Reinstein, J.; Vetter, I. R.; Schlichting, I.; Roesch, P.; Wittinghofer, A.; Goody, R. S. Fluorescence and NMR Investigations on the Ligand Binding Properties of Adenylate Kinases. Biochemistry 1990, 29, 7440 7450 . 